\title{
USO DE INTERNET PARA EVALUAR EL DISTRÉS PSICOLÓGICO EN PACIENTES CON CÁNCER DE MAMA
}

\section{USE OF INTERNET TO ASSESS PSYCHOLOGICAL DISTRESS IN BREAST CANCER PATIENTS}

\author{
Tania Estapél, Jordi Estapél, Sara Soria-Pastorl y Anna Díez ${ }^{2}$ \\ ' FEFOC, Barcelona, España, \\ ${ }^{2}$ Instituto de Medicina Predictiva i Personalizada del Cáncer (IMPPC), Badalona, España.
}

Resumen

Objetivo: El objetivo de este estudio es determinar la prevalencia de síntomas de distrés y la estrategia de adaptación entre una amplia muestra de pacientes de habla hispana con cáncer de mama mediante el uso de internet.

Métodos: Se diseñó un estudio transversal con pacientes con cáncer de mama $(n=434)$ reclutadas a través de Internet (http://www. cancermama.org). Las participantes completaron un cuestionario demográfico y médico, y también se usaron cuestionarios de evaluación psicológica ampliamente utilizados en Psicooncología, la HADs (Escala de Ansiedad y Depresión Hospitalaria) y la escala MAC (La Escala de Ajuste Mental al Cáncer), en su versión reducida de 29 ítems (Mini-MAC).

Resultados: En nuestro estudio el resultado global de la HADs como medida de distrés en línea es confiable en nuestro estudio. La tasa de prevalencia general de distrés para nuestra muestra fue del $50 \%$, sin diferencias según nivel socio-demográfico o variables médicas. En cuanto a puntuación de ansiedad $66,2 \%$ y $34 \%$ alcanzaron nivel clínico de ansiedad y depresión, respectivamente. Estos resultados se relacionaron con los de la Mini-MAC según lo esperado.

Conclusiones: Estos resultados dan apoyo al uso de Internet como herramienta válida para la evaluación de los trastornos psicológicos en pacientes con cáncer de mama. Internet nos brinda la posibilidad de identificar

\section{Abstract}

Objective: The object of this study is to determine the prevalence of distress symptoms and coping strategy among a large sample of Spanish-speaking breast cancer patients recruited on-line.

Methods: We designed a cross-sectional study with breast cancer survivors $(n=434)$ that were accrued via Internet (http://www. cancermama.org). Participants completed a demographic and medical questionnaire, and they were also evaluated using self-reporting measures of psychological distress and adjustment to cancer; the 14-item Hospital Anxiety and Depression Scale (HADS) and the 29-item version of the Mental Adjustment to Cancer Scale (Mini-MAC), respectively.

Results: Global HAD as a measure of distress on-line is reliable in our study. The overall prevalence rate of distress for our sample was $\mathbf{5 0} \%$, with no differences for sociodemographics or medical status variables. Concerning to anxiety punctuation $66.2 \%$ were clinical cases and $34.5 \%$ reached the clinical level for depression. These results were associated with Mini-MAC scales as expected.

Conclusions: These results offer support for the use of Internet as valid tool for the psychological distress assessment in patients with breast cancer Spanish-speaking patients. Internet brings us the possibility to identify highrisk patients through psychological screening in order to provide early interventions, and 
pacientes de alto riesgo a través de screening psicológico con el fin de realizar intervenciones tempranas, y las puntuaciones globales de distrés pueden ser una herramienta precisa. Sin embargo se necesitan más estudios para analizar la situación de consulta en internet como un estímulo provocador de ansiedad.

Palabras clave: Cáncer de mama, HADS, Mini-MAC, distrés, internet.
Global HAD punctuation may be an accurate tool. However more studies are needed to analyze internet illness consulting situation as an anxious provoking space.

Key words: Breast cancer, HADS, MiniMAC, distress, online assessment.

\section{INTRODUCCIÓN}

Internet es un nuevo recurso en varios aspectos de la vida. Salud y enfermedad son, con mucho, los temas que más se buscan en la navegación por internet. $Y$ las enfermedades que más se consultan en búsqueda de información son cáncer y $\operatorname{SIDA}^{(1,2)}$. En Oncología internet se utiliza cada vez más, no solo para obtener información general sobre la enfermedad ${ }^{(2-4)}$, sino también para temas relacionados con el tratamiento, el soporte y rehabilitación. La participación interactiva de los usuarios de Internet está creciendo en diferentes aspectos de la enfermedad, con espacios de asesoramiento médico y psicológico, foros, chats y ahora el uso de redes sociales con fines de búsqueda de apoyo e intercambio de experiencias $^{(2,5-8)}$. Es fácil, pues, entender que la psicooncología sea un tema habitual entre los relacionados con el cáncer que se buscan en la red. A pesar de que la investigación en psicooncología a través de Internet es todavía un enfoque novedoso, algunos estudios ya han utilizado la tecnología para evaluar síntomas de ansiedad, calidad de vida y el procesamiento emocional en la adaptación al cáncer de mama ${ }^{(9-11)}$. Asimismo, algunos grupos han empezado a usar facebook y otros canales habituales para trabajos en Psicooncología ${ }^{(5)}$.

Los avances en la tecnología informática y de Internet han hecho factible y atractiva la evaluación electrónica, como método de recolección de datos e información sobre los pacientes ${ }^{(12)}$. Además, como Jim y Andersen ${ }^{(11)}$ señalan, Internet también puede proporcionar una oportunidad a algunos individuos en situaciones especiales que, de otro modo no podrían participar en la investigación. Es decir que otra ventaja de este canal de investigación es que permite la mayor accesibilidad de pacientes con más dificultades de autonomía o desplazamiento, o en momentos de debilidad o deterioro físico debido al tratamiento.

Un pequeño pero creciente cuerpo de literatura sugiere cada vez más pruebas de la eficacia de la ayuda obtenida a través de Internet en pacientes con cáncer ${ }^{(13,14)}$. Esto no se refiere solo al soporte psicológico profesional, sino incluso al apoyo entre las propias pacientes (lo que vendría a ser los grupos de autoayuda). Algunos trabajos han Ilevado a cabo estudios controlados respecto a los beneficios de este tipo de intercambios entre las mujeres ${ }^{(15,16)}$.

El uso de la red no solo es para fines asistenciales y de apoyo e información sino también de investigación pues, como Schmidt y Andrykowski ${ }^{(9)}$ nos señalan permite recoger a un número mayor de muestras de los pacientes más rápidamente de lo que hubiera sido posible a través de los métodos tradicionales de muestreo. Es lo que viene a denominarse el "big data". Se ahorran costes y tiempo y se puede facilitar el acceso de muchos pacientes que vía presencial nunca hubieran participado ${ }^{(17)}$. 
También hay que valorar el tema de la evaluación psicológica. Debemos conseguir modelos evaluativos probados al igual que tenemos en el trato presencial, pues es la manera de determinar si la ayuda que proporcionamos es eficaz. Diversos estudios usan Internet para la evaluación de pacientes con cáncer. Manning et al.(10), con una muestra de enfermas con cáncer de mama reclutada a través de Internet, señalaron que casi todas las mujeres (98\%) estaban experimentando al menos un síntoma de distrés, sin diferencias significativas entre las mujeres que estaban en plena terapia adyuvante y los que ya la habían completado. Jim y Andersen ${ }^{(11)}$ llevaron a cabo dos estudios con supervivientes de cáncer con muestras recolectadas a través de Internet. Los participantes completaron medidas de funcionamiento físico y social, del significado de la enfermedad y el distrés. Los autores concluyeron que las secuelas negativas sociales $y$ físicas del cáncer se asocian con mayor distrés, y esta asociación parece ser explicada, en parte, por la pérdida de los pacientes de significado en sus vidas. Algunos estudios, como el de Steel et al. ${ }^{(6)}$ emplean cuestionarios estandarizados de calidad de vida que son de uso habitual en oncología. Todo esto nos indica un incremento del uso de Internet como algo paralelo al mundo presencial desde el cual cada vez más se pueden hacer aproximaciones al paciente oncológico.

\section{EVALUACIÓN DEL DISTRÉS}

La National Cancer Comprehensive Cancer Network (NCCN) definió en 1999 el distrés como las reacciones emocionales y psicológicos hacia el diagnóstico y tratamiento del cáncer, y desde entonces, varios estudios han centrado sus conclusiones en esta categoría ${ }^{(18-20)}$. El hablar de reacciones psicológicas al tratamiento del cáncer parece más exacto que la concep- ción habitual de ansiedad y depresión. El Distrés como variable objetivo de evaluación en pacientes con cáncer es habitual hoy en día, siendo un gran ejemplo el trabajo de Zabora et al. que ha evaluado una amplia muestra de pacientes con cán$\operatorname{cer}^{(21)}$. El termómetro de distrés parece ser el más ampliamente utilizado para obtener una evaluación numérica ${ }^{(19,22)}$. Sin embargo, algunos investigadores han sugerido el uso de la puntuación global de la HADs como una manera de obtener resultados de distrés. HADs es una herramienta habitual en el enfoque psicológico a pacientes con cáncer en la práctica clínica y en la investigación $^{(23,24)}$. La ansiedad y la depresión como categorías separadas han sido cuestionadas en la evaluación de pacientes con cáncer, y el uso de ambas como un resultado único parece ser un mejor enfoque para la medición psicológica en oncología ${ }^{(19)}$.

\section{WEB DE CÁNCER DE MAMA}

Nuestra fundación sin ánimo de lucro fue creada en 1996 con el fin de proporcionar información y apoyo a los pacientes y sus familiares y a la educación pública y la formación de profesionales. Internet apareció desde el principio como una herramienta paralela a nuestras actividades presenciales y su uso ha crecido. Hoy en día contiene cuatro webs vinculadas a la principal (www.fefoc.org). Éstas conforman nuestras principales áreas de trabajo más sólidas, es decir: cáncer de mama (www.cancermama.org), cáncer de próstata (www.cancerdeprostata.org), oncogeriatría (www.mayoressincancer) y familiares de pacientes (www.familiaycancer.org). Desde el principio se incluyó actividad interactiva, ofreciendo información médica mediante consultorio y segunda opinión. Con el tiempo esto ha crecido dadas las demandas y hoy día ha aumentado con consultas de tipo psicológico y emocional, legales, estéticas, de nutrición y muchos 
otros aspectos. La web de cáncer de mama es la más visitada en relación a los aspectos psicológicos, mientras que la de cáncer de próstata quizás crece más en la consulta de informaciones más médicas, seguramente por una cuestión de diferencias de género respecto a la enfermedad, como ya hemos presentado en otros foros ${ }^{(2,25,26)}$.

Desde 2004 se incluyeron tanto en la web de cáncer de mama como en la de próstata, no solo información sino la oportunidad de auto-evaluación de aspectos psicológicos para los visitantes. Esta medición incluye un breve formulario en datos socio-demográficos y de la enfermedad y los pacientes registrados pueden rellenar las escalas $\operatorname{HAD}^{(27,28)}$ y Mini-MAC en versión española ${ }^{(29,30)}$.

La razón para elegir Mini-MAC en lugar de la escala completa MAC es para evitar que la gente se canse y deja cuestionarios incompletos, que es uno de los riesgos de mediciones virtuales. Al terminar, los pacientes reciben respuesta inmediata en base a la línea de corte de la HAD y Mini-MAC. Esta respuesta es automática e informativa cuando el paciente Ilega al caso clínico se recomienda la búsqueda de apoyo psicológico.

Desde el principio esta evaluación psicológica apareció mucho más utilizada por las pacientes con cáncer de mama ${ }^{(2,20,25)}$. Es por eso que elegimos este ejemplo para analizar los niveles de estrés y los primeros resultados en nuestro proyecto de trabajo en línea.

\section{MÉTODO}

\section{Muestra / participantes}

Se realizó un diseño descriptivo transversal con una muestra obtenida a través de Internet. El acceso al sitio web utilizado para el reclutamiento de Internet está abierto pero los participantes deben registrarse mediante la proporción de sus datos para tener acceso a los cuestionarios del estudio.
El periodo de estudio fue de noviembre de 2007 hasta febrero de 2013. El criterio de inclusión fue que las pacientes se hubieran registrado en nuestra web de cáncer de mama y tener, como mínimo, 18 años de edad. Los datos son confidenciales y se registran en la ley de protección de datos. Las pacientes son informadas al respecto. Asimismo las pacientes. En ese tiempo 474 mujeres accedieron al protocolo pero 40 no siguieron respondiendo. Así pues, para el presente estudio se reclutó una muestra de 434 participantes en la web de cáncer de mama que antes hemos mencionado, entre noviembre de 2007 y febrero de 2013.

\section{Medidas}

Como dijimos antes se usó un cuestionario de datos socio-demográficos de las pacientes (las variables analizadas fueron: edad, estado civil, maternidad, nivel de estudios y situación laboral). Las variables biomédicas incluidas en esta parte del cuestionario fueron: tipo de cirugía (mastectomía o tumorectomía) y tiempo transcurrido desde la cirugía (más de un mes, entre uno y seis meses, entre seis y doce meses, de uno a tres años y tres años o más) y tratamientos complementarios recibidos después de la cirugía (incluyendo quimioterapia, radioterapia, terapia hormonal y todas sus combinaciones posibles).

El distrés psicológico se evaluó utilizando la HADs ${ }^{(23)}$. La HADS es un cuestionario de 14 ítems con dos dimensiones (depresión y ansiedad), que ha sido previamente validado como un instrumento adecuado para su uso con pacientes de cáncer españoles ${ }^{(27)}$. Una puntuación total igual o superior a 15 es indicativa de malestar clínicamente significativo(28).

\section{Escala Mini-MAC}

El Mini-MAC es la versión revisada y abreviada de una escala previa, la escala 
$\mathrm{MAC}^{(29)}$ (Watson et al., 1988) que cuenta con versión española ${ }^{(30)}$. Es una escala ampliamente usada en pacientes con cáncer y consta de 40 ítems. Los propios autores desarrollaron posteriormente una versión reducida de la misma escala para facilitar su aplicación y asegurar al máximo la recogida de resultados. Así se consiguió la escala Mini-MAC ${ }^{(31)}$ que consta de 29 ítems i es tipo Likert (4 puntos). Esta cuenta también con la versión española ${ }^{(32)}$. Las estrategias de afrontamiento evaluadas son cinco: Desesperanza/desamparo, que sería la propensión una actitud de pesimismo respecto al cáncer; Preocupación Ansiosa, que, tal como dice su propio nombre evalúa ansiedad y tensión ante el cáncer; Espíritu de Lucha, que se refiere a la que exhiben las pacientes que aceptan la enfermedad como un desafío y se muestran dispuestas a luchar activamente contra ella; Evitación Cognitiva o negación, que es la tendencia a evitar afrontar cognitivamente la enfermedad y lo que supone y Fatalismo, que es una sensación de falta de control ante la enfermedad y al mismo tiempo una tendencia a la resignación ${ }^{(29,32)}$.

\section{Procedimiento}

Las mujeres completaron los cuestionarios de autoinforme que se incluyen en este orden: un formulario de datos demográficos y clínicos, la HADs y la Mini-MAC.

\section{Análisis estadístico}

En primer lugar, se analizaron los datos mediante la prueba Kolmogorov-Smirnov, para determinar si seguían la distribución normal. Cuando los datos no cumplían los requisitos de normalidad se usaron aproximaciones no paramétricas para comparar las variables, test de $U$ de Mann-Whitney, $\mathrm{H}$ de Kruskal-Wallis. Asimismo se utilizaron coeficientes de correlación de Pearson o de Spearman para evaluar las asociaciones en- tre la HADs y las subescalas de la MiniMAC, según correspondiera. Todos los análisis estadísticos se realizaron utilizando el paquete estadístico para Ciencias Sociales (SPSS) versión 17.0. (SPSS Inc, Chicago, IL).

\section{RESULTADOS}

La frecuencia de rechazo fue del 3\%, esto es, pacientes que accedieron a los cuestionarios y no completaron el estudio, abandonando antes de empezar.

Las características socio-demográficas y clínicas de los pacientes se presentan en la Tabla 1. La edad media de esta muestra es de 43,86 años (DT= 8,9) siendo la participante más joven de 21 años y la mayor de 78. La mayoría de ellas están casadas o en pareja $(73,5 \%)$ y $68,2 \%$ tienen hijos. En cuanto al nivel educativo, la mayoría de esta muestra se concentra entre grado de secundaria $(24,7 \%)$ y estudios universitarios $(24,9 \%)$, también una alta proporción de este grupo de mujeres eran activas laboralmente en el momento de contestar a los cuestionarios (43,3\%).

En cuanto a las variables médicas 26 , $3 \%$ fueron diagnosticadas entre uno y tres años antes del participar en el estudio, siendo ésta es la categoría más frecuente relativa al tiempo transcurrido desde la cirugía. El segundo fue de entre uno y seis meses $(21,4 \%)$, seguidos por las mujeres recientemente diagnosticadas (un mes, el $18 \%)$, más de tres años $(18,9 \%$ ) y entre seis y doce meses $(15,4 \%)$. Cincuenta $y$ cinco por ciento de la muestra habían sido sometidas a cirugía radical (mastectomía) y el otro $44,9 \%$ a cirugía conservadora. En relación con el tratamiento seguido tras la cirugía, 25, 6\% había recibido quimioterapia (CT), 10,1\% terapia hormonal (TH), 9,4\% radioterapia (RT) y el $24 \%$ la combinación de estos tres regímenes terapéuticos. Otras combinaciones fueron de dos en dos como sigue: 18,7\% $\mathrm{CH}+\mathrm{RT}, 7,1 \% \mathrm{CT}+\mathrm{HT}$, la de menor fre- 
Tabla 1. Datos médicos y socio-demográficos $(\mathbf{n}=\mathbf{4 3 4})$

\begin{tabular}{|c|c|c|}
\hline & \\
\hline & $\mathbf{N}$ & $\%$ \\
\hline \multicolumn{3}{|l|}{ Datos socio-demográficos } \\
\hline $\begin{array}{l}\text { Edad } \\
<25 \\
25-35 \\
36-46 \\
47-57 \\
58-68 \\
69-78 \\
\text { Edad media (DE) }\end{array}$ & $\begin{array}{l}417 \\
4 \\
71 \\
193 \\
127 \\
19 \\
3 \\
43,86\end{array}$ & $\begin{array}{l}96,1 \\
1,0 \\
17,0 \\
46,3 \\
30,4 \\
4,6 \\
0,7 \\
(8,9)\end{array}$ \\
\hline $\begin{array}{l}\text { Estado civil } \\
\text { Casada/en pareja } \\
\text { Soltera } \\
\text { Divorciada/separada } \\
\text { Viuda } \\
\text { Otros }\end{array}$ & $\begin{array}{l}434 \\
319 \\
55 \\
42 \\
16 \\
2 \\
\end{array}$ & $\begin{array}{l}100 \\
73,5 \\
12,7 \\
9,7 \\
3,7 \\
0,5 \\
\end{array}$ \\
\hline $\begin{array}{l}\text { Maternidad } \\
\text { Si } \\
\text { No }\end{array}$ & $\begin{array}{l}434 \\
296 \\
138 \\
\end{array}$ & $\begin{array}{l}100 \\
68,2 \\
31,8\end{array}$ \\
\hline $\begin{array}{l}\text { Nivel de estudios } \\
\text { Primaria } \\
\text { Medio } \\
\text { Universitario } \\
\text { Otros }\end{array}$ & $\begin{array}{l}263 \\
17 \\
107 \\
108 \\
31\end{array}$ & $\begin{array}{l}60,6 \\
3,9 \\
24,7 \\
24,9 \\
7,1\end{array}$ \\
\hline $\begin{array}{l}\text { Situación laboral } \\
\text { Activ } \\
\text { De baja } \\
\text { Jubilada } \\
\text { Incapacidad } \\
\text { otros }\end{array}$ & $\begin{array}{l}434 \\
188 \\
132 \\
19 \\
18 \\
77 \\
\end{array}$ & $\begin{array}{l}100 \\
43,3 \\
30,4 \\
4,4 \\
4,1 \\
17,7 \\
\end{array}$ \\
\hline \multicolumn{3}{|l|}{ Datos médicos } \\
\hline $\begin{array}{l}\text { Tiempo desde la cirugía } \\
\text { Un mes } \\
\text { Entre uno y seis meses } \\
\text { Entre seis y doce meses } \\
\text { Entre uno y tres años } \\
\text { Más de tres años }\end{array}$ & $\begin{array}{l}434 \\
78 \\
93 \\
67 \\
114 \\
82\end{array}$ & $\begin{array}{l}100 \\
18,0 \\
21,4 \\
15,4 \\
26,3 \\
18,9\end{array}$ \\
\hline $\begin{array}{l}\text { Opción quirúrgica } \\
\text { Mastectomía } \\
\text { Tumorectomía }\end{array}$ & $\begin{array}{l}434 \\
239 \\
195 \\
\end{array}$ & $\begin{array}{l}100 \\
55,1 \\
44,9\end{array}$ \\
\hline $\begin{array}{l}\text { Opción post-quirúrgica } \\
\text { Quimioterapia } \\
\text { Radioterapia } \\
\text { Hormonoterapia } \\
\text { Quimioterapia y Hormonoterapia } \\
\text { Quimioterapia y Radioterapia } \\
\text { Radioterapia y Hormonoterapia } \\
\text { Qumioterapia, Hormonoterapia y Radioterapia }\end{array}$ & $\begin{array}{l}434 \\
111 \\
41 \\
44 \\
31 \\
81 \\
22 \\
104\end{array}$ & $\begin{array}{l}100 \\
25,6 \\
9,4 \\
10,1 \\
7,1 \\
18,7 \\
5,1 \\
24,0 \\
\end{array}$ \\
\hline
\end{tabular}


cuencia fue la combinación RT + HT que representa el 5,1\% de la muestra.

La tabla 2 muestra la prevalencia clínica de casos de ansiedad, depresión y también de las subescalas Mini-MAC, de acuerdo con el punto de corte habitual. La valoración como caso clínico de las estrategias de afrontamiento evaluadas implica una combinación entre puntuaciones bajas de espíritu de lucha y altas de desamparo-desespero. Las frecuencias se distribuyeron de la siguiente manera: 215 pacientes $(66,2 \%)$ en relación a la puntuación de ansiedad, $34,5 \%$ alcanzaron el nivel clínico para la depresión y 50,5\% para distrés. En cuanto a la escala Mini MAC, 3,5\% de la muestra exhibieron un espíritu de lucha bajo combinado con un estilo más alto de Desamparo más Desesperanza, 17,9\% fueron considerados como caso clínico referente a la escala preocupación ansiosa, 18,2\% en la evitación cognitiva y 7,5\% en fatalismo. La fiabilidad de la HADs como medida de distrés a través de internet se puso a prueba mediante el Alfa de Cronbach $(\alpha=0,894)$.

El análisis de los resultados globales de la HADs por variables socio-demográficas y médicas no mostró diferencias significativas. Aunque nuestro objetivo era analizar el distrés como variable objetivo, realizamos los mismos métodos para las subescalas de la Mini-MAC. Así pues, Fatalismo fue estadísticamente significativa (Mann-Whitney $U=-2,38, p<0,017$ ) por opción quirúrgica y espíritu de lucha por estado civil (KruskallWallis $\mathrm{H}=10,370, \mathrm{p}<0,035)$. Los resultados se muestran en la tabla 3.

Tal y como se expone en la tabla 3, generamos la variable distrés como variable objetivo, con dos categorías: "caso clínico" "no caso clínico". Se analizó esta variable por comparación de medias mediante el t-test para cada variable socio-demográfica y médica incluida en el estudio. Sólo tres variables socio-demográficas aparecieron como estadísticamente significativas por tener o no distrés (situación laboral, $\mathrm{F}=4,7$, $p<0,031$; estado civil, $F=7,77, p<0,006$ y maternidad, $F=9,04, p<0,003)$. Ninguna de las variables médicas aparecieron como relacionadas con esta variable.

Las correlaciones entre la HADs y las subescalas de la Mini-MAC se detallan en la tabla 4. Las medidas de la HADs (ansiedad, depresión y distrés) correlacionaron con todas las subescalas Mini-MAC, con excepción de evitación cognitiva. Se hallaron altas correlaciones significativas entre puntuaciones de ansiedad, depresión y la global de distrés y las subescalas de desesperanza y preocupación ansiosa de la Mini-MAC, según lo esperado por trabajos previos.

\section{Tabla 2. Prevalencia clínica de la HADs $(\mathbf{N}=341)$ y la Mini-MAC $(\mathbf{N}=\mathbf{3 0 9})$}

\begin{tabular}{|l|l|l|l|l|l|l|l|}
\cline { 2 - 8 } \multicolumn{1}{c|}{} & \multicolumn{3}{c|}{ HADs N (\%) } & \multicolumn{3}{c|}{ Mini-MAC N (\%) } & Ansiedad \\
\cline { 2 - 8 } & Depresión & Distrés & $\begin{array}{c}\text { Desamparo- } \\
\text { Desespero } \\
\text { y Espíritu } \\
\text { de Luchab }\end{array}$ & $\begin{array}{c}\text { Preocupación } \\
\text { ansiosa }\end{array}$ & $\begin{array}{c}\text { Evitación } \\
\text { cognitiva }\end{array}$ & Fatalismo \\
\hline Casos clínicos $^{\mathrm{a}}$ & $215(66,2)$ & $115(34,5)$ & $163(50,5)$ & $10(3,5)$ & $51(17,9)$ & $54(18,2)$ & $22(7,5)$ \\
\hline No casos clínicos & $110(33,8)$ & $218(65,5)$ & $160(49,5)$ & $274(96,5)$ & $234(82,1)$ & $242(81,8)$ & $271(92,5)$ \\
\hline $\begin{array}{l}\text { Total (sin valores } \\
\text { perdidos) }\end{array}$ & $325(95,3)$ & $333(97,7)$ & $323(94,7)$ & $284(91,9)$ & $285(92,2)$ & $296(95,8)$ & $293(94,8)$ \\
\hline
\end{tabular}

${ }^{a}$ Criterios de puntuación para considerar caso clínico: Subescalas de la HADs; Ansiedad y Depresión, puntuaciones $\geq 8$; Puntuaciones de Distrés $\geq 15$; Subescalas Mini-MAC; Puntuación Desamparo-Desespero y Espíritu de Lucha $<25$; Puntuación Preocupación Ansiosa $>24$; Puntuación Evitación $>12$; Puntuación Fatalismo $>17$. 'ba consideración de caso clínico para la MAC (y Mini-MAC) se obtiene a partir de la combinación entre puntuaciones bajas de espíritu de lucha y altas en desamparo-desespero. 


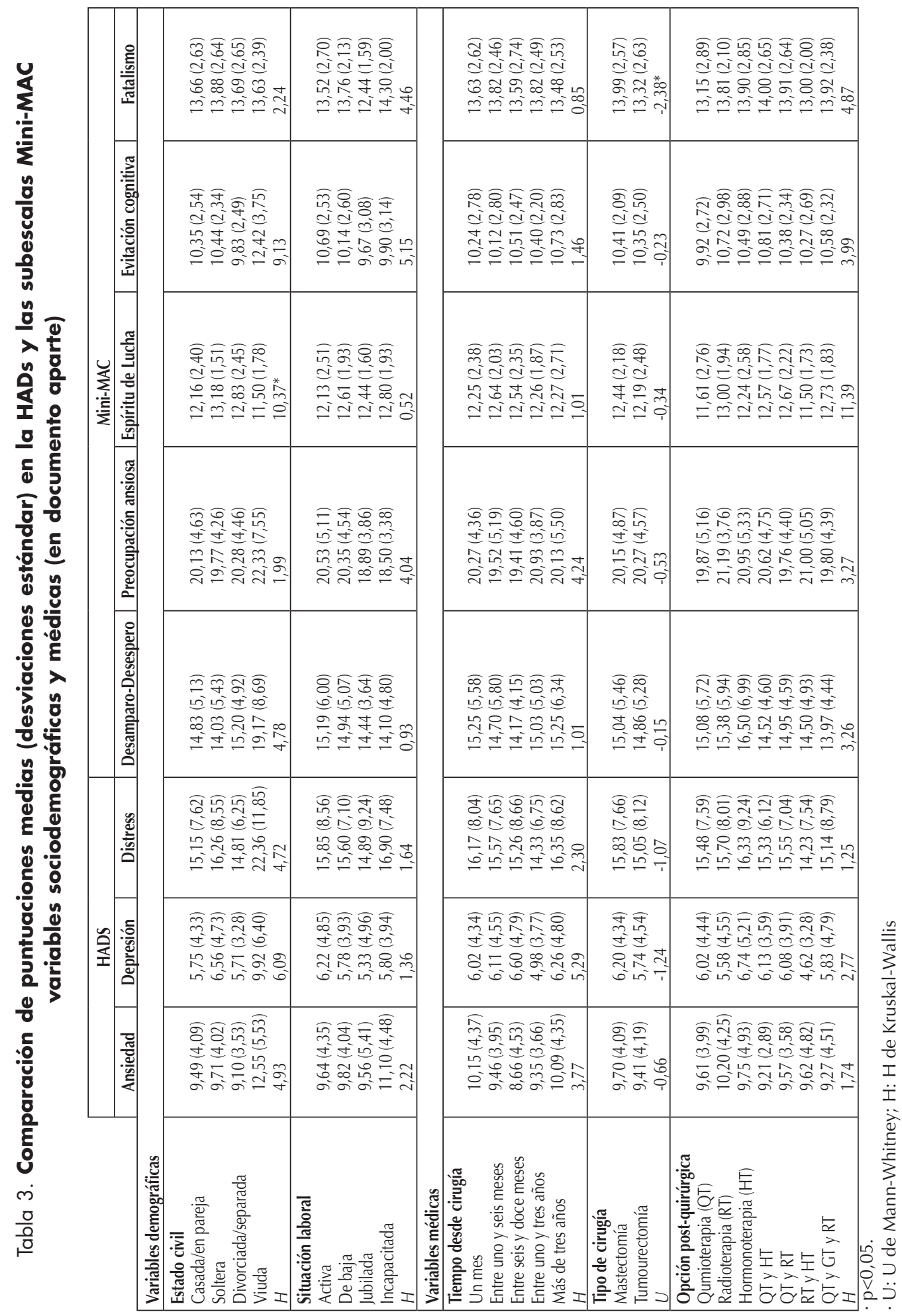


Al realizar correlaciones por separado por los dos instrumentos (tablas 5 y 6 ) se reportaron también resultados similares a lo que se espera: altas correlaciones entre la puntua- ción de distrés y ansiedad y depresión. También entre las subescalas de la Mini-MAC se hallaron altas correlaciones con asociación positiva o negativa según lo esperado.

\section{Tabla 4. Correlación entre subescalas HADs y Mini-MAC}

\begin{tabular}{|c|c|c|c|c|c|c|}
\hline & \multicolumn{5}{|c|}{ Mini-MAC } \\
\hline & & $\begin{array}{c}\text { Desemparo- } \\
\text { Desespero } \\
\text { Rho Spearman } \\
(p \text { valor })\end{array}$ & $\begin{array}{c}\text { Preocupación } \\
\text { Ansiosa } \\
\text { Pearson } \\
(p \text { valor }) \\
\end{array}$ & $\begin{array}{c}\text { Espíritu de } \\
\text { Lucha } \\
\text { Rho Spearman } \\
(p \text { valor })\end{array}$ & \begin{tabular}{|c|} 
Evitación \\
Cognitiva \\
Rho Spearman \\
$(p$ valor $)$
\end{tabular} & $\begin{array}{l}\text { Fatalismo } \\
\text { Rho } \\
\text { Spearman } \\
(p \text { valor })\end{array}$ \\
\hline \multirow{3}{*}{ HADS } & Ansiedad & $\begin{array}{c}0,158^{* *} \\
(0,008)\end{array}$ & $\begin{array}{c}0,233^{* *} \\
(0,001)\end{array}$ & $\begin{array}{c}-0,151^{*} \\
(0,12)\end{array}$ & $\begin{array}{l}-0,630 \\
(0,292) \\
\end{array}$ & $\begin{array}{c}-0,134^{*} \\
(0,026)\end{array}$ \\
\hline & Depresión & $\begin{array}{l}0,189 * * \\
(0,001)\end{array}$ & $\begin{array}{l}0,212^{* *} \\
(0,001)\end{array}$ & $\begin{array}{c}-0,191 * * \\
(0,001)\end{array}$ & $\begin{array}{c}0,050 \\
(0,399)\end{array}$ & $\begin{array}{c}-0,166^{* *} \\
(0,005)\end{array}$ \\
\hline & Distress & $\begin{array}{l}0,180^{* *} \\
(0,001)\end{array}$ & $\begin{array}{c}0,243^{* *} \\
(0,001)\end{array}$ & $\begin{array}{c}-0,177^{* *} \\
(0,003)\end{array}$ & $\begin{array}{l}-0,018 \\
(0,769)\end{array}$ & $\begin{array}{l}-0,149^{*} \\
(0,013)\end{array}$ \\
\hline
\end{tabular}

* Correlación significativa al nivel 0.05 (dos colas).

** Correlación significativa al nivel 0.01 (dos colas).

\section{Tabla 5. Coeficientes de la Correlación de Spearman (valores p) entre las subescalas de la HADs}

\begin{tabular}{|c|c|c|}
\cline { 2 - 3 } \multicolumn{1}{c|}{} & Depresión & Distrés \\
\hline \multirow{2}{*}{ Ansiedad } & $0,681^{* *}$ & $\begin{array}{l}0,915^{* *} \\
(0,001)\end{array}$ \\
\hline \multirow{2}{*}{ Depresión } & $(0,001)$ & $\begin{array}{l}0,913^{* *} \\
(0,001)\end{array}$ \\
\hline
\end{tabular}

** Correlación significativa al nivel 0,01 (dos colas).

Tabla 6. Correlaciones entre las subescalas de la Mini-MAC

\begin{tabular}{|c|c|c|c|c|c|}
\hline & \multicolumn{5}{|c|}{ Mini-MAC } \\
\hline & $\begin{array}{c}\text { Desemparo- } \\
\text { Desespero } \\
\text { Rho Spearman } \\
\quad(\text { valor } p)\end{array}$ & $\begin{array}{l}\text { Preocupación } \\
\text { Ansiosa } \\
\text { Pearson (valor } p \text { ) }\end{array}$ & $\begin{array}{c}\text { Espíritu de } \\
\text { Lucha } \\
\text { Rho Spearman } \\
\text { (valor } p \text { ) }\end{array}$ & $\begin{array}{c}\text { Evitación } \\
\text { Cognitiva } \\
\text { Rho Spearman } \\
(\text { valor } p)\end{array}$ & $\begin{array}{c}\text { Fatalismo } \\
\text { Rho Spearman } \\
\quad(\text { valor } p)\end{array}$ \\
\hline \multicolumn{6}{|l|}{ Mini-MAC } \\
\hline $\begin{array}{c}\text { Desemparo- } \\
\text { Desespero }\end{array}$ & --- & $\begin{array}{l}0,734^{* *} \\
(0,001)\end{array}$ & $\begin{array}{c}-0,502^{* *} \\
(0,001) \\
\end{array}$ & $\begin{array}{l}0,233^{* *} \\
(0,001) \\
\end{array}$ & $\begin{array}{c}-0,254^{* *} \\
(0,001) \\
\end{array}$ \\
\hline $\begin{array}{c}\text { Preocupación } \\
\text { Ansiosa }\end{array}$ & $\begin{array}{l}0,722^{* *} \\
(0,001) \\
\end{array}$ & --- & $\begin{array}{c}-0,264^{* *} \\
(0,001) \\
\end{array}$ & $\begin{array}{l}0,346^{* *} \\
(0,001)\end{array}$ & $\begin{array}{c}-0,184^{* *} \\
(0,001)\end{array}$ \\
\hline Espíritu de Lucha & $\begin{array}{c}-0,502^{* *} \\
(0,001) \\
\end{array}$ & $\begin{array}{c}-0,251^{* *} \\
(0,001) \\
\end{array}$ & --- & $\begin{array}{l}0,152^{* *} \\
(0,010)\end{array}$ & $\begin{array}{c}0,484^{* *} \\
(0,001)\end{array}$ \\
\hline $\begin{array}{l}\text { Evitación } \\
\text { Cognitiva }\end{array}$ & $\begin{array}{l}0,233^{* *} \\
(0,001) \\
\end{array}$ & $\begin{array}{l}0,346^{* *} \\
(0,001) \\
\end{array}$ & $\begin{array}{l}0,152^{*} \\
(0,010)\end{array}$ & --- & $\begin{array}{l}0,168^{* *} \\
(0,004)\end{array}$ \\
\hline Fatalismo & $\begin{array}{c}-0,254^{* *} \\
(0,001)\end{array}$ & $\begin{array}{c}-0,184^{* *} \\
(0,001)\end{array}$ & $\begin{array}{c}0,484^{* *} \\
(0,001)\end{array}$ & $\begin{array}{c}0,168^{* *} \\
(0,004)\end{array}$ & --- \\
\hline
\end{tabular}

* Correlación significativa al nivel 0,05 (dos colas).

** Correlación significativa al nivel 0,01 (dos colas). 


\section{DISCUSIÓN}

Se trata de un estudio preliminar para tratar de confirmar Internet como una forma válida y sólida para valorar el distrés psicológico entre pacientes de habla hispana con cáncer de mama. El porcentaje de rechazo es muy bajo si contabilizamos a las pacientes que decidieron acceder al estudio y luego abandonaron. Esto es sobretodo si lo comparamos con otros trabajos. Cull et al. ${ }^{(25)}$ por ejemplo, tenían inicialmente un $13 \%$ y un $5 \%$ en el estudio de seguimiento. No obstante la situación virtual, en nuestro trabajo, no puede dar información sobre los motivos reales para rechazar rellenar este cuestionario. Se les informa que recibirán retroalimentación inmediata sobre su distrés y la adaptación a la enfermedad, basada en los estilos de afrontamiento. En el futuro pensamos considerar la inclusión de un espacio con el fin de permitir que las pacientes expliquen las razones para no participar. Esto evitaría el sesgo psicosocial al rellenar cuestionarios por Internet. Creemos que esta es una de las debilidades de la línea psicooncológica que estamos usando. Nuestra muestra cuenta con un porcentaje elevado de mujeres con estudios superiores, lo que puede ser también un sesgo para algunos resultados. Otro estudio anterior en el que comparamos el perfil de la paciente con cáncer de mama usuaria de internet versus la no usuaria, ya arrojaba resultados sobre diferencias en el nivel académico(6).

Nuestro análisis indica que el uso de la medida global de distrés para las pacientes con cáncer de mama reclutados en línea es confiable, con una $\alpha=0,894$. Este resultado corrobora que este instrumento es apropiado para medir distrés al igual que en situaciones presenciales. Además, ésta variable correlaciona como se esperaba con las escalas de la Mini-MAC ${ }^{(26,29)}$.

No se encontraron diferencias en cuanto al distrés, en las variables socio-demográfi- cas ni médicas. La literatura indica diferencias en las reacciones emocionales ante el cáncer por edad. Se asume, por lo general, que ante el cáncer la edad tiene una relación inversa a la ansiedad, por lo que a más edad de la paciente menos ansiedad y viceversa ${ }^{(19,21)}$. En nuestro trabajo, observamos que nuestra muestra se acumula más en el grupo de edad mediana, que puede ser considerado joven teniendo en cuenta las edades en que el cáncer de mama es de más frecuente aparición (intervalos de 36 a 46 y 47 a 58 años) y una frecuencia muy baja del de mayor edad (sólo tres pacientes en el grupo de 69 a 78). Esta baja representatividad de los grupos de más edad puede explicar este resultado, o puede impedir conclusiones sólidas al respecto. Zabora et al. ${ }^{(21)}$ indica una disminución de la ansiedad a más edad del paciente, pero un aumento repentino en pacientes de mayor edad (después de 80 años de edad). Nuestra muestra tiene muy pocas pacientes de este grupo de mayor edad. A día de hoy, es un grupo que no utiliza Internet como una herramienta informativa o no usan Internet en absoluto ${ }^{(6)}$. Zabora et al. ${ }^{(21)}$ también analiza el nivel de ingresos como factor estresante posible, pero nosotros no hemos evaluado este aspecto de forma tan directa. Quizás lo más similar es nuestra aproximación a través del estatus profesional y laboral, pero no hemos hallado diferencias significativas según esta variable.

La muestra obtuvo un alto porcentaje en el nivel de ansiedad (66,2\%), depresión $(34,5 \%)$ y distrés $(50,5 \%)$ analizándolo bajo la perspectiva de caso clínico, o sea usando el punto de corte de 8 o más para cada una de las subescalas de la HADs. Para comparar con una muestra similar a la nuestra en características culturales, Grassi et al. ${ }^{(23)}$ han encontrado un $34 \%$ en ansiedad, $24,9 \%$ en depresión y $45,1 \%$ en distrés. Las diferencias son aún más elevadas cuando se toma la ansiedad y la depresión por separado. Este trabajo se realiza con diferentes tipos 
de cáncer, pero no se encontraron diferencias significativas al comparar el cáncer de mama con otras localizaciones. Los autores señalaron a un 49,1\% en el análisis del distrés de las pacientes con cáncer de mama. Usando una chi-cuadrado para este porcentaje, no se encontraron diferencias significativas con nuestra muestra $(\chi 2=0,037, \mathrm{p}=\mathrm{ns})$. Esto indicaría que el uso de la herramienta online es adecuada.

El alto porcentaje de ansiedad clínicamente significativa puede ser debida a la situación de "estar consultando" Internet. Las pacientes que están consultando una web que habla sobre su enfermedad en el momento pueden estar elicitando recuerdos sobre la misma y aumentando su ansiedad-estado, incluso si son supervivientes (intervalo 'más de tres años desde la cirugía'). Esto se puede considerar en el diseño de futuros trabajos de investigación en línea. Los estudios que citamos miden a los pacientes sin indicar el tiempo transcurrido desde el diagnóstico ${ }^{(30)}$. Pero el hecho de que esta variable en nuestro estudio no provoque diferencias significativas en la medida de repercusiones psicológicas es suficientemente importante como para analizarlo con cuidado. Sugerimos que dos factores podrían causar estos resultados. Por un lado, como decimos pensar en el momento en consulta de Internet como un momento ansioso. Las pacientes que están leyendo acerca de su enfermedad pueden sufrir reacciones emocionales en relación a esta situación, independientemente del tiempo transcurrido desde que fueron diagnosticadas. El otro factor que creemos que es probable como sesgo es plantear qué mujeres que ya han superado el cáncer hace cierto tiempo consultan internet y dentro de las mismas cuales pueden estar interesadas en interesadas en la auto-evaluación de su nivel de ansiedad. Esto sugiere una cierta consideración para futuros estudios: podría haber un sesgo difícil de definir dentro de la motivación de este grupo de mujeres.
Esto nos sugiere establecer una nueva investigación comparando estos resultados en línea con una muestra presencial que no consulte internet. Hemos comenzado este objetivo con la participación de tres servicios de oncología. Esto puede dar información adicional sobre la función de la situación de internet. Por ejemplo en un trabajo anterior comparando las repercusiones del cáncer de mama entre una muestra usuaria de internet y otra que no lo era, se mostraron algunas diferencias significativas, una de ellas, como dijimos antes, la edad, siendo más jóvenes los que llenan el cuestionario por internet ${ }^{(6)}$. Esto podría ser una buena manera de analizar o evitar el sesgo ansiedad debido a la situación de Internet. También en el futuro se podrían incluir medidas de ansiedad rasgo o estado (como la escala STAI por ejemplo). Cabe añadir que las supervivientes de cáncer que rellenan la autoevaluación quizás están bajo un momento especial (percepción de síntomas, cercanía de pruebas o visitas de control).

Otro aspecto a tener en cuenta es el sesgo en función de sus necesidades de obtener información o de ser anónimas, lo que puede indicar un perfil concreto. No sabemos los motivos que impelen a la mujer a consultar internet en ese momento y en qué situación se encuentran a nivel personal y emocional.

Nosotros pensamos que Internet es una buena herramienta para el acercamiento psicooncológico para pacientes que viven lejos de los hospitales o centros de atención primaria, para gente que prefiere el anonimato y para personas que están limitadas en sus desplazamientos debido a largas distancias, trabajo o situación física $^{(2,8,19)}$. También es una manera fácil de ahorro de costes para evaluar y proporcionar asesoramiento psicológico. La gran muestra que visita nuestra web, participa de espacios interactivos y pide consejo médico o psicológico indica que esta es una línea de trabajo que debemos seguir. No 
obstante, al igual que en la atención presencial, debemos conseguir instrumentos y tratamientos probados, para promover una Psicooncología basada en la evidencia y ofrecer lo mejor a nuestros pacientes.

\section{Agradecimientos}

Damos las gracias a todos los pacientes por su participación voluntaria en este estudio. También damos las gracias a la Dra. Maggie Watson por brindar toda su ayuda en el uso de la escala Mini-MAC y por darnos permiso para utilizarla en nuestro trabajo clínico y de investigación y también a Silvia Juanes por su ayuda en el trabajo estadístico.

\section{REFERENCIAS BIBLIOGRÁFICAS}

1. Risk A, Dzenowagis J. Review of internet health information quality initiatives. J Med Internet Res 2001;3:E28. Doi:10.2196/ jmir.3.4.e28

2. Estapé T, Estapé J, Boixadós M, Alberro JA. How do spanish-speaking breast cancer patients who use internet differ from those who do not use? Psychooncology 2006; 15 (Suppl 2): 234-5.

3. Friedman DB, Hoffman-Goetz L, Arocha JF. Readability of cancer information on the internet. J Cancer Educ 2004;19:11722. Doi:10.1207/s15430154jce1902_13

4. Unruh HK, Bowen DJ, Meischke $\mathrm{H}$, Bush N, Wooldridge JA. Women Health 2004;40:59-78. Doi:10.1300/ J013v40n01_04

5. Scanlon K, Carol R. The role of facebook and twitter in signposting. Psychooncology 2013;22 (Suppl 3): 16. Doi: 10.1111/ j.1099-1611.2013.3393

6. Steel J, Kim K, Geller D, Brower D, Philips Ch, Ordos J, Marsh W, Tsung A. A Webbased collaborative care intervention for patients with advanced cancer. Psychooncology 2013;22 (Suppl.3):1-123. Doi: 10.1111/j.1099-1611.2013.3393
7. Bach PB. Using practice guidelines to assess cancer care quality. J Clin Oncol 2005;23:9041-3.

8. Seale C. Portrayals of treatment decisionmaking on popular breast and prostate cancer web sites. Eur J Cancer Care 2005;14:171-4. Doi:10.1111/j.13652354.2005.00544.x

9. Schmidt JE, Andrykowski MA. The role of social and dispositional variables associated with emotional processing in adjustment to breast cancer: an internetbased study. Health Psychol 2004;23:25966. Doi: 10.1037/0278-6133.23.3.259

10. Manning-Walsh J. Social support as a mediator between symptom distress and quality of life in women with breast cancer. J Obstet Gynecol Neonatal Nurs 2005;34:482-93. Doi: 10.1177/0884217505278310

11. Jim HS, Andersen BL. Meaning in life mediates the relationship between social and physical functioning and distress in cancer survivors. $\mathrm{Br}$ J Health Psychol 2007;12(Pt 3):363-81. Doi:10.1348/135910706X128278

12. Berry DL, Trigg LJ, Lober WB, Karras BT, Galligan ML, Austin-Seymour M, et al. Computerized symptom and quality-oflife assessment for patients with cancer part I: Development and pilot testing. Oncol Nurs Forum 2004;31:E75-83. Doi: 10.1188/04.ONF.E75-E83

13. Winzelberg A, Classen C, Alpers G, Roberts H, Koopman C, Adams R., et al. Evaluation of an Internet support group for women with primary breast cancer. Cancer 2003;97:1164-73. Doi:10.1002/cncr.11174

14. Klemm P, Bunnell D, Cullen M, Soneji R, Gibbons P, Holecek A. Online cancer support groups: A review of the research literature. Comput Inform Nurs 2003;21:136-42.

15. Salzer MS, Palmer SC, Kaplan K, Brusilovskiy E, Ten Have T, Hampshire M, et al. A randomized, controlled study of Internet peer-to-peer interactions among 
women newly diagnosed with breast cancer. Psychooncology 2010;19:441-6. Doi: 10.1002/pon.1586.

16. Eysenbach G, Powell J, Englesakis M, Rizo C, Stern A. Health related virtual communities and electronic support groups: Systematic review of the effects of online peer to peer interactions. B Med J 2004;328:1166-70. Doi: 10.1136/ bmj.328.7449.1166

17. Taenzer, P Bultz, BD Carlson, LE Speca, M DeGagne, T Olson, K Doll, R Rosberger, Z. Impact of computerized quality of life screening on physician behaviour and patient satisfaction in lung cancer outpatients. Psychooncology 2000;9:203-13. Doi:10.1002/1099$1611(200005 / 06) 9: 3<203:$ :AID PON453>3.0.CO;2-Y

18. National Comprehensive Cancer Network Barry D. Practice guidelines in oncology-v.1.2002: Distress management: National Comprehensive Cancer Network.

19. Carlson LE, Bultz BD. Cancer distress screening. Needs, models, and methods. J Psychosom Res 2003;55:403-9. Doi: 10.1016/S0022-3999(03)00514-2

20. Bultz BD, Carlson L. Emotional distress: The sixth vital sign: future directions in cancer care. Psychooncology 2006;15:93-5.

21. Zabora, J Brintzenhofeszoc, K Curbow, B Hooker, C Piantadosi,,S. The prevalence of psychological distress by cancer site. Psychooncology 2001;10:19-28.

22. Jacobsen PB, Donovan KA, Trask PC, Fleishman SB, Zabora J, Baker F, et al. Screening for psychologic distress in ambulatory cancer patients. Cancer 2005;103:1494-502. Doi: 10.1002/ cncr.20940

23. Grassi L, Travado, L Moncayo, FLG Sabato, S Rossi, E SEPOS Group. Psychosocial morbidity and its correlates in cancer patients of the Mediterranean area: Findings from the Southern European Psychooncology Study. J Affect Disord 2004;83:243-8. Doi:10.1016/j.jad.2004.07.004
24. Cull A, Gould A, House A, Smith A, Strong V, Velikova G, et al. Validating automated screening for psychological distress by means of computer touchscreens for use in routine oncology practice. $\mathrm{Br}$ J Cancer 2001;85:1842-9. Doi:10.1054/ bjoc.2001.2182

25. Estape T, Estape J. Psychological assessment by internet for Spanish and Latinoamerican patients. Psychooncology 2003;12(Suppl 4):S204.

26. Estape T, Estape J, Soria S. Use of internet in psychological assessment in breast cancer patients. Psychooncology 2008;17:S168-S168.

27. López-Roig S, Terol MC, Pastor Mira MA, Neipp MC, Massutí B, Rodríguez Marín J. Ansiedad y Depresión. Validación de la escala HAD en pacientes oncológicos. Rev Psicol Salud 2000;12:127-55

28. Ibbotson $T$, Maguire $P$, Selby $P$, Priestman T, Wallace L. Screening for anxiety and depression in cancer patients: The effects of disease and treatment. Eur J Cancer 1994;30A:37-40.

29. Watson M, Greer S, Young J, Inayat Q, Burgess C, Robertson B. Development of a questionnaire measure of adjustment to cancer: the MAC scale. Psychol Med 1988;18: 203-209. Doi: 10.1017/ S0033291700002026

30. Ferrero, J Barreto, MP Toledo, M. Mental adjustment to cancer and quality-of-life in breast-cancer patients - an exploratorystudy. Psychooncology 1994;3:223-32. Doi: 10.1002/pon.2960030309

31. Watson M, Law M, Dos Santos M, Greer S, Baruch J, Bliss, J. The Mini-MAC: Further development of the manual of The Mental Adjustment to Cancer Scale. J Psychosoc Oncol 1994;12:33-46. Doi:10.1300/ J077V12N03_03

32. Pérez Rodríguez S. Sintomatología de estrés postraumático en pacientes con cáncer de mama e identificación de posibles factores de riesgo [tesis doctoral]. Valencia: Universidad de Valencia; 2008 
